\section{REVISTA BRASILEIRA DE QUALIDADE DE VIDA}

\title{
Queixa vocal e qualidade de vida em voz de mulheres tabagistas
}

\section{Vocal complaints and quality of life in voice of women smokers}

\author{
Vanessa Ribeiro \\ Universidade Estadual do Centro-Oeste/UNICENTRO - Irati - Paraná - Brasil \\ vanessaribeirinha@ hotmail.com \\ Vanessa Veis Ribeiro \\ Universidade Estadual do Centro-Oeste/UNICENTRO - Irati - Paraná - Brasil \\ vanessaribeirooo@hotmail.com \\ Ana Paula Dassie-Leite \\ Universidade Estadual do Centro-Oeste/UNICENTRO - Irati - Paraná - Brasil \\ pauladassie@hotmail.com
}

\section{RESUMO}

OBJETIVO: Verificar a presença de queixa vocal e os índices de qualidade de vida em voz de mulheres tabagistas.

MÉTODOS: A amostra foi composta por 36 mulheres, tabagistas, com idades entre 19 e 44 anos. Aplicou-se um questionário contendo dados de identificação, histórico em relação ao tabagismo e queixas vocais e o protocolo Qualidade de Vida em Voz (QVV). Os dados foram analisados estatisticamente por meio de testes não-paramétricos Mann-Whitney e Correlação de Spearman, adotando-se o nível de significância de 5\%.

RESULTADOS: O tempo médio de consumo de cigarro foi 17,69 anos, consumindo-se em média 16,33 cigarros/dia. 30,56\% das mulheres apresentaram queixa vocal. A média obtida no protocolo QVV para o grupo geral foi de 95,83 no domínio socioemocional, 91,55 no domínio físico e 93,13 no domínio total. No entanto, as mulheres que possuíam queixas vocais tiveram escores mais baixos nos domínios socioemocional e total do protocolo QVV.

CONCLUSÕES: Conclui-se que mulheres tabagistas que apresentam queixas vocais, mesmo com média de idade de 32,83 anos, possuem índices baixos de qualidade de vida relacionada à voz, compatíveis com os apresentados por indivíduos disfônicos.

PALAVRAS-CHAVE: Voz. Distúrbios da voz. Disfonia. Qualidade de vida.

\section{ABSTRACT}

OBJECTIVE: Verify the quality of life in women smokers' voice.

METHODS: The sample consisted of 36 women, smokers, aged 19 to 44 years. We applied a questionnaire containing identification data regarding smoking history and vocal complaints, and the protocol for Voice Related Quality of Life (V-RQOL). Data were analyzed statistically using 
non-parametric tests of the Mann-Whitney and Spearman correlation, adopting a significance level of $5 \%$.

RESULTS: The average cigarette consumption was 17,69 years, consuming on average 16,33 cigarettes/day. $30,55 \%$ of women had vocal complaints. The mean score on the protocol for VRQOL for the general group was 95,83 in socioemotional domain, 91,55 in the physical domain and 93,13 in the total domain. However, women who had vocal complaints had lower scores in the socio-emotional domains and total V-RQOL protocol.

CONCLUSIONS: We conclude that female smokers who have vocal complaints, even with an average age of 32.83 years, have low rates of related quality of life voice, compatible with those presented by dysphonic.

KEYWORDS: Voice. Voice disorders. Dysphonia. Quality of life.

\section{Introdução}

O tabagismo é considerado um problema de saúde pública que acomete cerca de um terço da população mundial, correspondendo a um bilhão e duzentos milhões de usuários (PEGANI; SOUZA; PEGANI, 2007). No Brasil, há cerca de 36,5 milhões de fumantes (AMORIM-FILHO et al., 2003).

O tabaco é utilizado há muitas décadas, principalmente por indivíduos do sexo masculino. O consumo entre as mulheres, com a conquista de espaços sociais e inserção efetiva no mercado de trabalho, ganha proporção significativa a partir da década de 60 (AMORIM-FILHO et al., 2003; PEGANI; SOUZA; PEGANI, 2007). Atualmente, cerca de 40,4\% dos usuários de tabaco são mulheres (AMORIM-FILHO et al., 2003).

A utilização do tabaco pode levar o sujeito a desenvolver desde problemas respiratórios até doenças crônicas não degenerativas (REINALDO; GOECKING; GOULART, 2010). Além da saúde geral, a fumaça do tabaco também é agressiva ao trato vocal, podendo levar ao aparecimento de alterações laríngeas como hiperemia, inflamação do trato vocal, laringite crônica, carcinoma laríngeo, além do eritema e do edema. Além disso, o tabagismo é o principal fator de risco para o desenvolvimento do câncer de laringe (VASCONCELOS et al., 2009; CIELO et al., 2010).

As queixas vocais mais frequentes nesses indivíduos são ardor, sensação de aperto na garganta, tosse, pigarro e aumento de secreção. Quanto à produção vocal, observam-se características específicas como redução dos tempos máximos de fonação (TMF), ataque vocal brusco, alterações de pitch e de loudness (CIELO et al., 2010; BEHLAU et al., 2001). Quanto à qualidade vocal, essa pode caracterizar-se por conter rugosidade, soprosidade, ou as duas características associadas (CAMARGO, 2003; FIGUEIREDO et al., 2003).

As alterações vocais causadas pelo tabagismo podem interferir negativamente na autoavaliação da qualidade de vida relacionada à voz dessa população (BEHLAU et al., 2009). A literatura atual traz vários protocolos traduzidos e validados para o português que são bastante sensíveis e tem como objetivo investigar o impacto de uma alteração vocal no cotidiano do sujeito, dentre os quais se destaca o protocolo Qualidade de Vida em Voz (QVV) (BEHLAU et al., 2009; GASPARINI; BEHLAU, 2009), que vem sendo amplamente utilizado na literatura para quantificar a percepção do indivíduo acerca da influencia da voz na sua vida.

$\mathrm{Na}$ literatura não foram encontrados estudos que avaliassem a qualidade de vida relacionada à voz de mulheres tabagistas. Acredita-se que tais dados possam ser importantes para que o clínico conheça a percepção vocal dessa população, auxiliando no direcionamento das campanhas de promoção de saúde vocal.

Sendo assim, o objetivo do presente estudo foi verificar a presença de queixa vocal e os índices de qualidade de vida em voz de mulheres tabagistas. 


\section{Métodos}

Trata-se de um estudo descritivo e transversal, de carácter observacional, aprovado pelo Comitê de Ética em Pesquisa da Universidade Estadual do Centro-Oeste sob o parecer nº52/2011. A pesquisa foi realizada segundo os preceitos do Conselho Nacional de Ética em Pesquisa (CONEP), estabelecidos na resolução $n^{\circ} 196 / 96$. Os participantes receberam os esclarecimentos necessários sobre o estudo e assinaram o Termo de Consentimento Livre e Esclarecido (TCLE).

Foram considerados critérios de inclusão na pesquisa: possuir idade mínima de 18 anos; apresentar bom estado de saúde geral; ter consumido ao menos 100 cigarros e ainda fazer uso dele, de acordo com os critérios para que o indivíduo seja considerado tabagista (WHO, 1983). Os critérios de exclusão adotados foram: ter diagnóstico de patologias vocais diversas (fendas, alterações estruturais mínimas, nódulos, entre outras), relacionadas a diferentes etiologias que não o tabagismo, no passado ou presente; já ter passado por tratamento fonoterapêutico e/ou otorrinolaringológico devido a problema de voz; estar no período de menopausa; estar em crise alérgica e/ou respiratória no dia da coleta; fazer uso profissional da voz; apresentar queixa de refluxo laringofaríngeo; ter histórico de quaisquer outras doenças que pudessem interferir negativamente na autopercepção vocal (neurológicos, psiquiátricos, oncológicos, auditivos e respiratórios).

Dos 50 sujeitos voluntários interessados em participar da pesquisa, excluiu-se: 9 pela faixa etária, 4 por utilizarem a voz profissionalmente e 1 pelo número de cigarros consumidos. Sendo assim, a amostra constituiu-se de 36 mulheres que responderam a um questionário (contendo dados de identificação e do histórico em relação ao tabagismo e presença ou não de queixa vocal) e ao protocolo de autoavaliação vocal QVV.

O QVV é um protocolo composto por dez itens que abrangem dois domínios: o de funcionalidade física e o domínio socioemocional. $\mathrm{O}$ instrumento é composto por uma escala de 1 a 5 , que indica a frequência que determinado evento ocorre. Cada afirmativa tem como possível resposta e pontuação: 1 - nunca acontece e não é um problema; 2 - acontece pouco e raramente é um problema; 3 - acontece às vezes e é um problema moderado; 4 - acontece muito e quase sempre é um problema; 5 - acontece sempre e realmente é um problema ruim. O protocolo tem como função a autoavaliação vocal ligada à análise dos aspectos de qualidade de vida relacionados à voz. Ainda, oferece um escore total (variando de 0 a 100, em que 0 indica pior qualidade de vida e 100 melhor qualidade de vida) e um escore para cada domínio (GASPARINI; BEHLAU, 2009; HOGIKYAN; SETHURAMAN, 1999; TUTYA et al., 2011). O protocolo possui um cálculo específico para transformar os escores em uma escala de percentuais (GASPARINI; BEHLAU, 2009).

Os dados foram tabulados. As variáveis foram analisadas estatisticamente por meio de testes não paramétricos Mann-Whitney e Correlação de Spearman. Foi adotado o nível de significância de $5 \%$, ou seja, todos os intervalos de confiança do trabalho foram construídos com $95 \%$ de confiança estatística.

\section{Resultados}

A amostra foi constituída por 36 sujeitos do sexo feminino, tabagistas, com idades entre 19 e 44 anos, média de idade de 32,83 anos. O tempo de consumo de cigarro variou entre 1 e 25 anos, e a média foi 17,69 anos. Com relação à quantidade de cigarros consumidos por dia, observou-se que a média foi de 16,33 cigarros, variando entre 1 e 50 cigarros/dia.

Quanto à presença de queixa vocal, 11 mulheres relataram ter queixa relacionada à voz $(30,56 \%)$ e 25 mulheres $(69,44 \%)$ não possuíam queixa, com diferença significativa $(p=0,001)$.

Os escores médios de qualidade de vida relacionados à voz de mulheres tabagistas foram menores no escore físico, seguido pelo total e socioemocional $(91,55 ; 93,13 ; 95,83$, respectivamente) (Tabela 1$)$. 
Tabela 1 - Analise descritiva dos escores do QVV em mulheres tabagistas

\begin{tabular}{llccc}
\hline & \multicolumn{1}{c}{ Domínios } & Média & Mediana & Desvio Padrão \\
\hline \multirow{4}{*}{ QVV } & Sócio-emocional & 95,83 & 100,00 & 11,67 \\
& Físico & 91,55 & 95,80 & 11,63 \\
& Total & 93,13 & 16,25 & 10,42 \\
\hline
\end{tabular}

Análise descritiva.

Legenda: QVV=qualidade de vida em voz

Fonte: Autoria própria (2014).

Na Tabela 2 é possível visualizar que mulheres com queixas vocais possuem escores médios mais baixos de qualidade de vida relacionada à voz nos domínios socioemocional $(p=0,002)$ e total $(\mathrm{p}=0,001)$.

Tabela 2 - Associação entre queixa e com domínios do QVV

\begin{tabular}{|c|c|c|c|c|}
\hline Protocolo & Domínios & Queixa Voc & Média & p-valor \\
\hline \multirow{6}{*}{ QVV } & \multirow{2}{*}{ Sócio-emocional } & Sim & 88,06 & \multirow{2}{*}{$0,002 *$} \\
\hline & & Não & 99,25 & \\
\hline & \multirow{2}{*}{ Físico } & Sim & 84,45 & \multirow{2}{*}{0,009} \\
\hline & & Não & 94,68 & \\
\hline & \multirow{2}{*}{ Total } & Sim & 85,45 & \multirow{2}{*}{$0,001 *$} \\
\hline & & Não & 96,51 & \\
\hline
\end{tabular}

* Valores estatisticamente significantes ( $\mathrm{p} \leq 0,05)$ - Teste Mann-Whitney

Legenda: QVV=qualidade de vida em voz

Fonte: Autoria própria (2014).

Não foi encontrada correlação entre os domínios de qualidade de vida relacionada à voz e a quantidade de cigarros consumidos por dia (Tabela 3) e o tempo de tabagismo (Tabela 4).

Tabela 3 - Correlação entre quantidade de cigarros consumidos/dia e os domínios do QVV

\begin{tabular}{clll}
\hline Quantidade de Cigarros consumidos/dia & Domínios & Coeficiente & p-valor \\
\hline \multirow{2}{*}{ QVV } & Sócio-emocional & $-0,059$ & 0,729 \\
& Físico & 0,248 & 0,143 \\
& Total & 0,049 & 0,772 \\
\hline
\end{tabular}

* Valores estatisticamente significantes $(\mathrm{p} \leq 0,05)$ - Correlação de Spearman

Legenda: QVV=qualidade de vida em voz

Fonte: Autoria própria (2014).

Tabela 4 - Correlação entre tempo de tabagismo/anos e os domínios do QVV.

\begin{tabular}{clll}
\hline Tempo de Tabagismo/anos & \multicolumn{1}{c}{ Domínios } & Coeficiente & p-valor \\
\hline \multirow{3}{*}{ QVV } & Sócio-emocional & $-0,059$ & 0,729 \\
& Físico & $-0,056$ & 0,741 \\
& Total & $-0,008$ & 0,960 \\
\hline
\end{tabular}

* Valores estatisticamente significantes $(\mathrm{p} \leq 0,05)$ - Correlação de Spearman

Legenda: QVV=qualidade de vida em voz

Fonte: Autoria própria (2014).

\section{Discussão}

O tabagismo é um hábito deletério altamente agressivo para o trato vocal, trazendo consequências graves que vão desde alterações laríngeas histopatológicas até o câncer de laringe (ALVARENGA et al., 2008; VIEIRA; BEHLAU, 2009). A voz do sujeito tabagista também assume 
características específicas, podendo ou não influenciar na qualidade de vida do indivíduo, dependendo da acuracia de sua autopercepção (CIELO et al., 2010; BEHLAU et al., 2001).

Os escores médios do protocolo QVV encontrados no grupo de mulheres tabagistas $(95,83$ no socioemocional; 91,55 no físico e 93,13 no total) estão muito próximos aos recomendados na literatura para indivíduos não disfônicos, sendo eles: 99,30 pontos no escore socioemocional, 98,00 pontos no físico e 97,10 no total (BEHLAU et al., 2009; GASPARINI; BEHLAU, 2009).

Mulheres com queixas vocais apresentaram escores menores nos domínios socioemocional e total do protocolo QVV. Acredita-se que os indivíduos que tem queixas relacionadas à voz possuem também uma autopercepção vocal mais acurada dos sinais e sintomas vocais no seu dia-a-dia. Esses achados concordam com os encontrados na literatura em um trabalho que aplicou o protocolo QVV em 97 professores da rede municipal, divididos em dois grupos: com queixas vocais $(61,20 \%)$ e sem queixas $(38,80 \%)$, onde os grupos apresentaram boa qualidade de vida em voz, mas com índices inferiores para os indivíduos com queixa vocal (MARTINELLO, 2009). Esse estudo reforça os achados da presente pesquisa, sugerindo ligação entre a percepção dos sintomas vocais e a qualidade de vida relacionada à voz. Tais dados mostram que os sintomas vocais influenciam na qualidade de vida, e acredita-se que influencie também na procura dos sujeitos por assistência profissional (CHIO-CARDIM; BEHLAU; ZAMBON, 2010; MUSIAL et al., 2011).

O escore físico do protocolo QVV pode não ter sido diferente entre indivíduos que possuem ou não queixas vocais devido ao número relativamente pequeno de sujeitos na amostra, uma vez que o valor de p obtido $(0,009)$ indica tendência à diferença. É possível que, se a amostra fosse maior, este domínio também mostrasse diferença entre as mulheres com e sem queixas.

$\mathrm{Na}$ presente pesquisa, mulheres tabagistas adultas, a quantidade de cigarros consumidos diariamente e o tempo de tabagismo não se correlacionaram aos escores de qualidade de vida em voz. Acredita-se que pelo fato de a média de idade das participantes ser baixa (32,83 anos), muitas delas ainda não tenham sentido o impacto negativo causado pelo cigarro na laringe e, principalmente, nas pregas vocais. Além disso, a média de tempo de tabagismo também foi menor do que a apontada por estudo anterior (SALES et al., 2006), e, por isso, pode ainda não estar causando sinais e sintomas vocais em intensidade perceptível e incômoda para as mulheres.

As mulheres tabagistas pesquisadas não eram profissionais da voz. Sendo assim, não possuíam grande demanda vocal no dia-a-dia, o que pode ter contribuído para que a maioria não tenha relatado queixas vocais e apresentassem altos índices de qualidade de vida relacionada à voz. A literatura vem mostrando que indivíduos que possuem grande demanda vocal como os profissionais da voz, possuem uma autopercepção vocal mais acurada, e costumam apresentar resultados diferentes dos indivíduos que não possuem tal demanda (GRILLO; PENTEADO, 2005; PUTNOKI et al., 2010; SPINA et al., 2009). Isso ocorre porque o impacto da disfonia na qualidade de vida do sujeito depende das características de vida e estilos individuais, fazendo com que, muitas vezes, não aja uma relação direta entre o prognóstico da alteração vocal e sua qualidade de vida (PARK; BEHLAU, 2011). O fato das participantes referirem boas condições de saúde também pode ter contribuido para os altos índices de qualidade de vida relacionada à voz, e a pouca ocorrência de queixas vocais.

Em suma, embora a maior parte das mulheres tabagistas ter referido ausência de queixas e de impacto negativo na qualidade de vida relacionada à voz, aproximadamente 30,56\% já mencionam queixas que podem estar relacionadas ao tabagismo. Tais queixas são acompanhadas de baixos escores de qualidade de vida em voz (índices compatíveis com os apresentados por indivíduos disfônicos) e merecem ser observados e valorizados pelos profissionais de saúde. Com o passar do tempo e com as modificações hormonais enfrentadas pelas mulheres durante a menopausa (MACHADO; ALDRIGHI; YUNES, 2003), as queixas vocais podem aumentar e os escores de qualidade de vida relacionada à voz podem diminuir, influenciando negativamente no dia-a-dia dessas mulheres. Em estudos futuros, deve-se contemplar mulheres com idade e tempo de consumo de tabaco mais elevados, a fim de que se trace um perfil acerca da ocorrência das queixas e distúrbios vocais nessa população. 


\section{Considerações finais}

Conclui-se que mulheres tabagistas que apresentam queixas vocais, mesmo com média de idade de 32,83 anos, possuem índices baixos de qualidade de vida relacionada à voz, compatíveis com os apresentados por indivíduos disfônicos.

\section{Referências}

ALVARENGA, L. M.; RUIZ, M. T.; PAVARINO-BERTELLI, E. C.; RUBACK, M. J. C.; MANIGLIA, J. V.; GOLONI-BERTOLLO, E. M. Avaliação epidemiológica de pacientes com câncer de cabeça e pescoço em um hospital universitário do noroeste do estado de São Paulo. Revista Brasileira de Otorrinolaringologia, v. 74, n. 1, p. 68-73, jan./fev. 2008. rossef

AMORIM-FILHO, S. A.; ANDRADE-SOBRINHO, J.; RAPOPORT, A.; CARVALHO, M. B.; NOVO, N. F.; JULIANO, Y. Estudo de variáveis demográficas, ocupacionais e co-carcinogenéticas no carcinoma espinocelular da base de língua nas mulheres. Revista Brasileira de Otorrinolaringologia, v. 69, n. 4, p. 472-478, jul./ago. 2003.

BEHLAU, M.; MADAZIO, G.; FEIJÓ, D.; PONTES, P. Avaliação de voz. In: BEHLAU, M. (Org.). Voz: o livro do especialista. Rio de Janeiro: Revinter; 2001.

BEHLAU, M.; OLIVEIRA, G.; SANTOS, L. de M. A. dos; RICARTE, A. Validação no Brasil de protocolos de auto-avaliação do impacto de uma disfonia. Pró-Fono Revista de Atualização Científica, v. 21, n. 4, p. 326-232, out./dez. 2009. rossef

CAMARGO, Z. Avaliação objetiva da voz. In: CARRARA-DE-ANGELIS, E. (Org.). A atuação da fonoaudiologia no câncer de cabeça e pescoço. São Paulo: Lovise; 2003.

CHIO-CARDIM, K.; BEHLAU, M.; ZAMBON, F. Sintomas vocais e perfil de professores em um programa de saúde vocal. Revista CEFAC, v. 12, n. 5, p. 811-819, 2010. rrossef

CIELO, C. A.; FINGER, L. S.; ROMAN-NIEHUES, G.; DEUSCHLE, V. P.; SIQUEIRA, M. A. Hábitos de tabagismo e etilismo em disfonias. Revista de Ciências Médicas e Biológicas, v. 13, n. 2, p. 119-125, 2010.

FIGUEIREDO, D. C.; SOUZA, P. R. F.; GONÇALVES, M. I. R.; BIASE, N. G. Análise perceptivo-auditiva, acústica computadorizada e laringológica da voz de adultos jovens fumantes e não fumantes. Revista Brasileira de Otorrinolaringologia, v. 69, n. 6, p. 791-799, nov./dez. 2003. crossef

GASPARINI, G.; BEHLAU, M. Quality of life: validation of the Brazilian version of the VoiceRelated Quality of Life (V-RQOL) Measure. Journal of Voice, v. 23, n. 1, p. 76-81, 2009. Crossef

GRILLO, M. H. M. M.; PENTEADO, R. Z. P. Impacto da voz na qualidade de vida de professore(a)s do ensino fundamental. Pró-Fono Revista de Atualização Científica, v. 17, n. 3, p. 321-330, set./dez. 2005. rossef

HOGIKYAN, N. D.; SETHURAMAN, G. Validation of an instrument to measure voice-related quality of life (V-RQOL). Journal of Voice, v. 13, p. 4, p. 557-569, 1999. 
MACHADO, M. A. M. P.; ALDRIGHI, J. M.; YUNES, J. Voz e mudanças vocais após a menopausa. Revista da Associação Médica Brasileira, v. 49, n. 3, p. 225-243, 2003. rossef

MARTINELLO, J. Avaliações psicométricas de qualidade de vida e voz em professores da rede municipal de Bauru. 2009. 161 f. Dissertação (Mestrado em Fonoaudiologia) - Programa de PósGraduação em Fonoaudiologia, Universidade de São Paulo, Bauru, 2009.

MUSIAL, P. L.; DASSIE-LEITE, A. P.; ZABOROSKI, A. P.; CASAGRANDE, R. C. Interferência dos sintomas vocais na atuação profissional de professores. Distúrbios da Comunicação, v. 23, n. 3, p. 335-341, 2011.

PARK, K.; BEHLAU, M. Sinais e sintomas da disfunção autônoma em indivíduos disfônicos. Jornal da Sociedade Brasileira de Fonoaudiologia, v. 23, n. 2, p. 164-169, 2011.

PEGANI, C. R.; SOUZA, E. G.; PEGANI, T. C. S. O tabagismo nos dias atuais. Ensaios e Ciência, v. 2, n. 2, p. 116-122, 2007.

PUTNOKI, D. S.; HARA, F.; OLIVEIRA, G. G.; BEHLAU, M. Qualidade de vida em voz: o impacto de uma disfonia de acordo com sexo, idade e uso vocal profissional. Revista da Sociedade Brasileira de Fonoaudiologia, v. 15, n. 4, p. 485-490, 2010. Crossef

REINALDO, A. M. S.; GOECKING, J. P. A.; GOULART, Y. N. Uso de tabaco entre adolescentes: revisão de literatura. Revista Eletrónica Salud Mental Alcohol y Drogas, v. 6, n. 2, p. 350-364, 2010.

SALES, M. P.; FIGUEIREDO, M. R.; OLIVEIRA, M. I.; CASTRO, H. N. Outpatient smoking cessation program in the state of Ceará, Brazil: patient profiles and factors associated with treatment success. Jornal Brasileiro de Pneumologia, v. 32, n. 5, p. 410-417, 2006. Crossef

SPINA, A. L.; MAUNSELL, R.; SANDALO, K.; GUSMÃO, R.; CRESPO, A. Correlação da qualidade de vida e voz com atividade profissional. Revista Brasileira de Otorrinolaringologia, v. 75 , n. 2, p. 275-279, 2009. rossef

TUTYA, A. S.; ZAMBON, F.; OLIVEIRA, G.; BEHLAU, M. Comparação dos escores dos protocolos QVV, IDV e PPAV em professores. Revista da Sociedade Brasileira de Fonoaudiologia, v. 16, n. 3, p. 273-281, 2011. Crossef

VASCONCELOS, S. V.; MELLO, R. J. V.; SILVA, H. J.; SOARES, E. B. Efeito do fumo nas dimensões das pregas vocais no idoso. International Archives of Otorhinolaryngology, v. 13, n. 1, p. 24-29, 2009.

VIEIRA, A. C.; BEHLAU, M. Análise de voz e comunicação de professores de curso prévestibular. Revista da Sociedade Brasileira de Fonoaudiologia, v. 14, n. 3, p. 346-351, 2009. crossef

WHO, World Health Organization. Guidelines for the conduct of tobacco smoking among health professionals. Report WHO. Meeting Winnipeg, Canadá. 1983. 